\section{Germline mosaicism for a GNAS1 mutation and Albright hereditary osteodystrophy}

EDITOR-Albright hereditary osteodystrophy (AHO) is an autosomal dominant disorder characterised by short stature, a round face with a low, flat nasal bridge, brachymetaphalangism, particularly of the fourth and fifth digits, obesity, subcutaneous calcification, and variable degrees of mental retardation. Patients may show end organ resistance to parathyroid hormone (PTH), known as pseudohypoparathyroidism type Ia (PHPIa). Patients with PHPIa may have hypocalcaemia, hyperphosphataemia, and raised PTH levels and show an absent or blunted cAMP response to exogenous PTH. ${ }^{1}$ Resistance to other hormones such as thyroid stimulating hormone (TSH) and luteinising hormone have also been reported. Other patients with AHO have a normal biochemical profile with a normal cAMP response to a PTH infusion, known as pseudopseudohypoparathyroidism (PPHP).

PHPIa and PPHP both result from inactivating mutations of the GNAS1 gene on chromosome 20. The PHPIa and PPHP phenotypes have been observed to segregate within the same family and thus represent variable expressivity of the same mutation. There is an apparent parent of origin effect: PHPIa usually results from maternal inheritance of a GNAS1 mutation while paternal transmissions give rise to offspring with PPHP. ${ }^{2}$ GNAS1 has 13 exons and mutations associated with AHO occur throughout the gene..$^{3-8}$ The new mutation rate at GNAS1 is unknown, but de novo occurrence of mutations has been proven in a number of families ${ }^{5-7}$ (our own unpublished observations). We now describe a family comprising two affected sibs whose presentation was complicated by gastrointestinal symptoms not previously associated with Albright hereditary osteodystrophy. Neither parent showed any features of AHO.

Patient 1 was born at term to non-consanguineous Cypriot parents. She developed persistent diarrhoea on the second day of life, resulting in episodes of dehydration, metabolic acidosis, and failure to thrive. At 4 months of age, her barium meal was normal and small bowel biopsy showed no focal abnormality. However, pancreatic function was markedly abnormal, with only a small volume of fluid recovered and low levels of lipase and trypsin. Neonatal thyroid screen was normal but endocrine investigations in the first year showed raised TSH and low growth hormone levels. She was thus started on thyroxine and hydrocortisone. Parathyroid hormone was found to be significantly raised at $230 \mathrm{pg} / \mathrm{ml}$ (normal range 10-70). She subsequently developed persistently low calcium levels and PTH infusion test showed no cyclic AMP response in plasma or urine, consistent with pseudohypoparathyroidism. She was started on 1- $\alpha$-hydroxy-D3 and calcium supplementation. Her growth has subsequently normalised and at $5 \frac{1}{2}$ years of age she now lies between the 50 th and 90th centile for weight and between the 10th and 50th centile for height. She remains dysmorphic with coarse facies, initially suggestive of mucopolysaccharidosis, and shows striking brachydactyly but no gross metacarpal or metatarsal shortening. She has approximately six months global developmental delay. Her initial problems with GI absorption have now settled and she no longer has evidence of pancreatic insufficiency.

Her younger brother, patient 2, was born following an uncomplicated pregnancy and delivery. $\mathrm{He}$ also showed loose stools from birth but gained weight more readily than his sister. Two small bowel biopsies performed at $2 \frac{1}{2}$ months of age showed mild enteropathy with some villus atrophy. Pancreatic function tests showed pancreatic insufficiency, which like his sister he eventually outgrew. Raised TSH was detected on neonatal thyroid screening and he was started on thyroxine. His PTH and calcium were initially within the normal range but in the second year of life he was found to have a low corrected calcium and significantly increased PTH (200). PTH infusion testing was not carried out. He also developed brachydactyly and coarse facies with a flattened nasal bridge and some frontal bossing. When last reviewed at $31 / 4$ years his small bowel biopsy was normal and there was no evidence of ongoing pancreatic insufficiency. He was found to have left optic atrophy of uncertain origin.

DNA was extracted from EDTA anticoagulated blood by standard methods. Individual exons of GNAS1 were amplified by polymerase chain reaction (primer sequences and PCR conditions available on request). Single stranded conformation analysis of exons 2, 3, 5, 7-10, 12, and 13 was carried out using $1 \times$ MDE acrylamide gels (Flowgen) run overnight at $4^{\circ} \mathrm{C}$ on a Protean II xi system (Biorad) followed by silver staining. ${ }^{9}$ An abnormal banding pattern suggestive of a heterozygous insertion was detected in exon 13 in DNA from patient 1. Sequence analysis using Big-Dye terminator chemistry (PE Biosystems) on an ABI 377 system confirmed the presence of a $12 \mathrm{bp}$ insertion (denoted 1107-1108insGCTGTGGACACT) in both affected sibs. The mutation is in frame and is predicted to result in tandem duplication of codons 366-369 inclusive. It was not detectable in lymphocyte DNA from either parent, both of whom were clinically unaffected. These results suggested parental germline mosaicism for a dominant mutation rather than recessive inheritance.

To investigate this further, DNA was obtained from two unaffected children in the family. The absence of the exon 13 mutation was confirmed in both. Linkage analysis was carried out using a FokI polymorphism in exon 5 of GNAS1 and a single nucleotide polymorphism in intron 12 (M A Aldred, unpublished data) together with flanking microsatellite markers D20S100, D20S171, and D20S173. Paternity was consistent for these chromosome 20 markers and also for seven microsatellite loci on other chromosomes. All four parental haplotypes could be determined. Haplotype analysis confirmed the presence of germline mosaicism and indicated that the mutation is maternal in origin (fig 1), consistent with the hormone resistant phenotype in the affected children.

The gastrointestinal problems of the affected children have not previously been associated with AHO. $\mathrm{G}_{\mathrm{s}} \alpha$ is ADP ribosylated by cholera toxin, an event which leads to constitutive activation of $\mathrm{G}_{\mathrm{s}}$ in the gut. The diarrhoea in these children is therefore not easily reconciled with the classical features of AHO that result from inactivating mutations. In addition, it has recently become clear that the enteropathy in patient 2 may result from cow's milk sensitivity and is therefore probably an unrelated finding. His pancreatic insufficiency may have been secondary to enteropathy. 

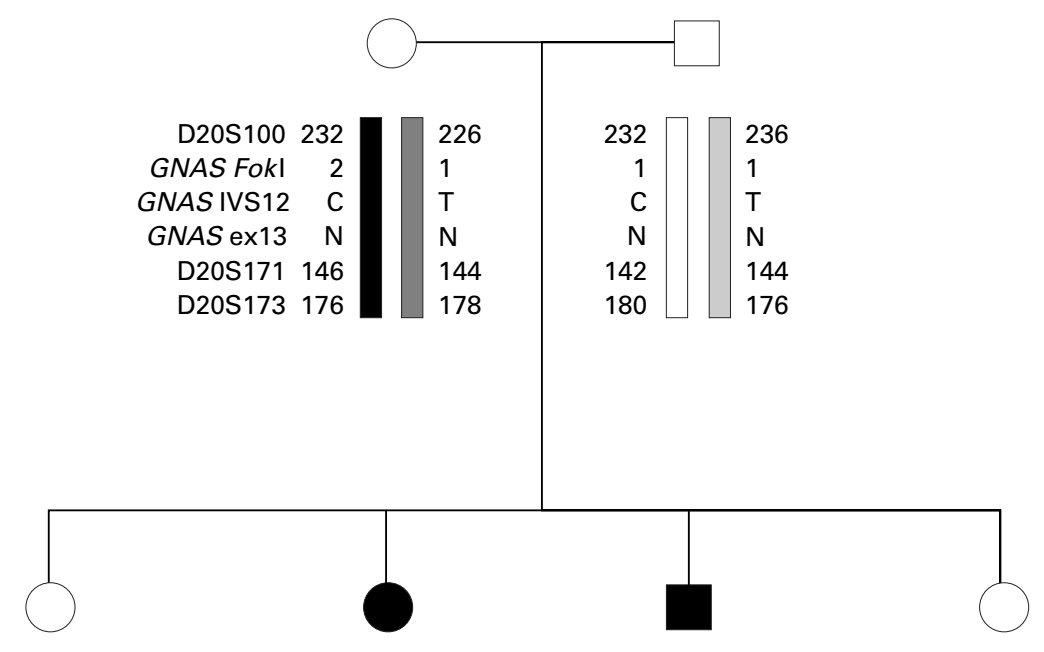

$\left.\begin{array}{rr}\text { D20S100 } & 236 \\ \text { GNAS FokI } & 1 \\ \text { GNAS IVS12 } & \mathrm{T} \\ \text { GNAS ex13 } & \mathrm{N} \\ \text { D20S171 } & ? \\ \text { D20S173 } & 176\end{array}\right] \square\left[\begin{array}{l}226 \\ 1 \\ \mathrm{~T} \\ \mathrm{~N} \\ ? \\ 178\end{array}\right.$
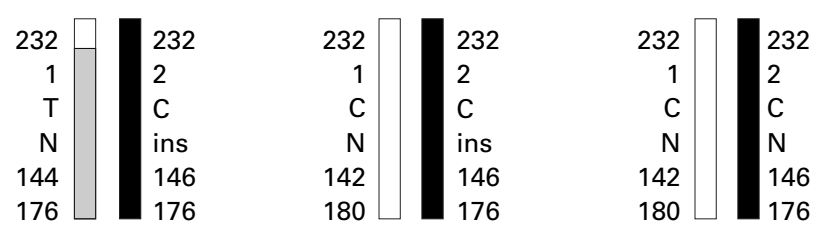

Figure 1 Results of the linkage analysis show that patients 1 and 2 have inherited a common maternal haplotype but no mutation was detectable in maternal lymphocyte DNA. One unaffected sib is haploidentical with patient 2 but does not carry the insertion mutation, thus confirming the presence of germline mosaicism. Order of microsatellite markers: cen-D20S100-11cM-D20S171-2.1cM-D20S173-tel. The most likely location for GNAS1 is between D20S100 and D20S173 but its location relative to D20S171 is not well defined. $N=$ normal, ins $=12$ bp insertion within exon 13 .

Nonetheless, the concordance in phenotype between the two affected children is remarkable and does suggest that some of these additional features could be an unusual manifestation of the GNAS1 mutation or result from a second, autosomal recessive, condition segregating in this family.

Pancreatic insufficiency has been reported in one other case of AHO.$^{10}$ The patient also had recurrent episodes of pneumonia and chronic constipation but investigations for cystic fibrosis were negative. Interestingly, the missense mutation identified in that patient was also in exon 13 of GNAS1, suggesting a possible genotype-phenotype correlation. The insertion mutation that we report occurs in a linker region between $\beta$ strand and $\alpha$ helix regions. It is also adjacent to one of five conserved sequences that form the nucleotide binding pocket. Functional analysis will be required to determine whether this insertion causes a simple loss of function or whether it produces a protein with altered function that may shed further light on the normal role of $\mathrm{G}_{\mathrm{s}} \alpha$.

Germline mosaicism has been documented in a number of genetic diseases with high new mutation rates, notably Duchenne muscular dystrophy, retinoblastoma, and tuberous sclerosis. There is a previous description of apparent recessive inheritance of AHO but the alternative explanation of mosaicism could not be excluded. ${ }^{11}$ Our analysis provides the first molecular confirmation of germline mosaicism in AHO.

Genetic counselling in apparently sporadic cases of $\mathrm{AHO}$ is problematical for a number of reasons. Firstly, the phenotype is highly variable, including a distinction between subjects showing hormone resistance (PHP) and those that are hormone responsive (PPHP). There is increasing evidence of a parent of origin effect, possibly because of imprinting of the GNAS1 locus, in determining hormone responsiveness in this condition. Secondly, the new mutation rate at the GNAS1 locus is unknown. We now add to this complexity with the molecular confirmation of germline mosaicism for a GNAS1 mutation. The frequency with which this may occur is at present unknown, but the possibility of germline mosaicism should now be considered when counselling recurrence risks to parents of a child with sporadic Albright hereditary osteodystrophy.

We thank Professor J O'Riordan, Middlesex Hospital for advice on interpretation of PTH infusion test results, Professor M Savage, St Bartholomew's Hospital for his advice concerning the management of initial growth failure, and Lindsey Sutherland for completing the microsatellite genotyping. RCT and Lindsey Sutherland for completing the microsatellite genotyping. RCT Childhood.

MICHEALA A ALDRED*†

R JANE BAGSHAW *

KAY MACDERMOT $\ddagger$

DAVID CASSONS

SIMON H MURCHS

J A WALKER-SMITHS

RICHARD C TREMBATH*+

${ }^{\star}$ Leicestershire Genetics Centre, Leicester Royal Infirmary, Leicester

LE1 $5 \mathrm{WW}, \mathrm{UK}$

†Division of Medical Genetics, Departments of Medicine and Genetics,

University of Leicester, Leicester LE1 7RH, UK

$\ddagger$ Department of Clinical Genetics, Royal Free Hospital, Pond Street,

London NW3 2QG, UK

§University Department of Paediatric Gastroenterology, Royal Free

Hospital, Pond Street, London NW3 2QG, UK

Correspondence to: Dr M A Aldred, maldred@hgmp.mrc.ac.uk

1 Tomlinson S, Hendy GN, O'Riordan JLH. A simplified assessment of response to parathyroid hormone in hypoparathyroid patients. Lancet 1976;i:62-4

2 Davies SJ, Hughes HE. Imprinting in Albright's hereditary osteodystrophy. f Med Genet 1993;30:101-3.

3 Patten JL, Johns DR, Valle D, Eil C, Gruppuso PA, Steele G, Smallwood PM, Levine MA. Mutation in the gene encoding the stimulatory $G$ protein of adenylate cyclase in Albright's hereditary osteodystrophy. $N$ Engl $\mathcal{F}$ Med 1990;322:1412-18.

4 Miric A, Vechio JD, Levine MA. Heterogeneous mutations in the gene encoding the $\alpha$-subunit of the stimulatory G protein of adenylyl cyclase in Albright hereditary osteodystrophy. F Clin Endocrinol Metabolism 1993;76: 1560-8.

5 Wilson LC, Oude Luttikhuis MEM, Clayton PT, Fraser WD, Trembath RC. Parental origin of $\mathrm{G}_{\alpha} \alpha$ gene mutations in Albright's hereditary osteodystrophy. F Med Genet 1994;31:835-9.

6 Oude Luttikhuis MEM, Wilson LC, Leonard JV, Trembath RC. Characterisation of a de novo 43-bp deletion of the $\mathrm{G}_{\mathrm{s}} \alpha$ gene (GNAS1) in Albright

7 Yu S, Yu D, Hainline BE, Brener JL, Wilson KA, Wilson LC, Oude Luttikhuis MEM, Trembath RC, Weinstein LS. A deletion hot-spot in exon 7 of the $\mathrm{G}_{\mathrm{s}} \alpha$ gene (GNAS1) in patients with Albright hereditary osteodystrophy. Hum Mol Genet 1995;4:2001-2. 
8 Shapira H, Mouallem M, Shapiro MS, Weisman Y, Farfel Z. Pseudohypoparathyroidism type Ia: two new heterozygous frameshift mutations in exons 5 and 10 of the $\mathrm{G}_{\mathrm{s}} \alpha$ gene. Hum Genet 1996;97:73-5.

9 Wallace AJ. Detection of unstable trinucleotide repeats. In: Elles R, ed. Molecular diagnosis of genetic diseases. Totowa, New Jersey: Humana Press, 1996:37-62
10 Schwindinger WF, Miric A, Zimmerman D, Levine MA. A novel G $\alpha$ mutant in a patient with Albright hereditary osteodystrophy uncouples cell

11 Cederbaum SD, Lippe BM. Probable autosomal recessive inheritance in a family with Albright's hereditary osteodystrophy and an evaluation of the genetics of the disorder. Am f Hum Genet 1973;25:638-45. 